Contract No. and Disclaimer:

This manuscript has been authored by Savannah River Nuclear Solutions, LLC under Contract No. DE-AC09-08SR22470 with the U.S. Department of Energy. The United States Government retains and the publisher, by accepting this article for publication, acknowledges that the United States Government retains a non-exclusive, paid-up, irrevocable, worldwide license to publish or reproduce the published form of this work, or allow others to do so, for United States Government purposes. 


\title{
Photoinduced Currents in CdZnTe Crystals as a Function of Illumination Wavelength
}

\author{
L C Teague ${ }^{1}$, A L Washington, II ${ }^{1}$, M C Duff ${ }^{1}$, M Groza ${ }^{2}$, V Buliga ${ }^{2}$, and A Burger ${ }^{2}$ \\ ${ }^{1}$ Savannah River National Laboratory, Aiken, South Carolina 29808, USA \\ ${ }^{2}$ Fisk University, Nashville, Tennessee 37208, USA
}

Email: lucile.teague@srnl.doe.gov

\begin{abstract}
We report variations in the currents of CdZnTe semiconductor crystals during exposure to a series of light emitting diodes of various wavelengths ranging from $470 \mathrm{~nm}$ to $950 \mathrm{~nm}$. The changes in the steady-state current of one CdZnTe crystal with and without illumination along with the time dependence of the illumination effects are discussed. Analysis of the de-trapping and transient bulk currents during and after optical excitation yield insight into the behaviour of charge traps within the crystal. Similar behaviour is observed for illumination of a second CdZnTe crystal suggesting that the overall illumination effects are not crystal dependent.
\end{abstract}

\section{Introduction}

CdZnTe (CZT) semiconducting crystals have a high atomic number and exhibit high bulk resistivity, which make them excellent candidates for room temperature hard $\mathrm{X}$ - and $\gamma$-ray spectrometers.[1] CZT semiconductors are also of interest for medical and space imaging, solar cells, and as substrates for the growth of other materials used for infrared detector applications.[14] Several factors can affect the charge transport and collection properties of these materials including the presence of defects and heterogeneities such as secondary phases (SPs).[5] Further study will help to delineate the contributions of these defects on the intrinsic electrical properties of CZT. A thorough understanding of the specific relationships between the structural, electronic and optical properties of CZT can help guide future material and application developments.

The charge trap distribution and electrical field distribution are relevant to CZT performance in many applications. Previous reports have utilized the Pockels effect in CZT (due to its zinc- 
blende crystal structure) to examine the spatial distribution of the electric field in CZT crystals.[610] One of these studies, by Sellin, et al., reports that the electric field distribution in CZT can be manipulated by controlling the crystal temperature.[7] Our recent study has shown that a similar, yet separate polarization effect is observed following sub-bandgap illumination of the CZT crystal.[8] While this study focused on the electric field distribution, herein, we examine the effects of illumination at multiple wavelengths on the bulk currents in two different CZT crystals grown under different conditions. These studies focused on the role of electron and hole traps in CZT to further our understanding of the complex charge transport dynamics in these ternary systems.

It is well established that there is a significant difference in hole and electron transport efficiency in CZT materials, with hole mobility lifetime products being as much as 10 fold lower than those of electrons. Charge traps due to impurities and defects, such as SPs [5] will further influence the charge transport through these crystals. Due to their slower mobility, holes are more easily trapped within the CZT crystal, while electrons are more weakly trapped and more evenly

distributed throughout the crystal due to their higher mobility.[11] Although several models have been proposed to correlate the specific effects of charge traps due to impurities and defects such as SPs, these effects are still not well understood.

\section{Experimental Details}

The two CZT crystals used for our studies, designated R64039B and YT-3-7-8, were grown with a $\sim 10 \%$ Zn content, and were acquired from Redlen Technologies (British Columbia, Canada) and Yinnel Tech (YT, South Bend, IL), respectively. The estimated band gap for CZT with this Zn content is $\sim 1.6 \mathrm{eV}$.[7] More information on these crystals has been published elsewhere.[5, 8, 12,13] The surfaces of each crystal were mechanically polished using standard techniques, with a final $0.05 \mu \mathrm{m}$ alumina polish. Gold $(\mathrm{Au})$ contacts were sputtered onto the as-polished crystal and the bulk currents were measured at room temperature as described below. 
The room temperature $\left(24.0 \pm 5^{\circ} \mathrm{C}\right)$ steady-state bulk current of $\mathrm{R} 64039 \mathrm{~B}$ was measured with applied electric fields of $\pm 78 \mathrm{~V} \mathrm{~mm}^{-1}$ and $\pm 133 \mathrm{~V} \mathrm{~mm}^{-1}$ in a Pockels setup (described elsewhere) $[6,8]$ to allow the simultaneous measurement of the bulk current and the electric field distribution. The changes in the electric field distribution at various wavelengths will be described in detail elsewhere. ${ }^{\mathrm{i}}$ For these measurements, Au contacts were sputtered on the crystal in planar configuration. The thin Au contact on the Te-rich CZT surface was held at ground and a single LED was positioned at a fixed distance $(\sim 38 \mathrm{~mm})$ above this crystal surface during the measurements with an estimated 36 to $49 \%$ light transmission through the contact. The LED light sources were as follows: a) $950 \mathrm{~nm}, 77 \mu \mathrm{W}$, b) $630 \mathrm{~nm}, 90 \mu \mathrm{W}, 722 \mu \mathrm{W}$ and c) $470 \mathrm{~nm}, 93 \mu \mathrm{W}$, $729 \mu \mathrm{W})$. A bias was applied to the Au contact on the Cd rich surface, opposite the light source. The Te and Cd rich surfaces ((111)B and (111)A, respectively) [14] of the crystal were previously identified via the methods described by Myers et al. [15] Distinctive features of the crystal with respect to these surfaces were noted to enable tracking of the Cd vs. Te rich surfaces, and the crystals were then polished as described above. The spectral emissions from the LEDs used are as follows: $950 \mathrm{~nm}( \pm 5 \mathrm{~nm}$ filter used $), 470 \mathrm{~nm}(\mathrm{FWHM}=25 \mathrm{~nm}$, no filter $)$ and 630 $\mathrm{nm}(\mathrm{FWHM}=25 \mathrm{~nm}$, no filter). Illumination conditions were as follows: the bias was applied to the crystal and the $1115 \mathrm{~nm}$ light was turned on at 101 seconds (s). The LED illumination $470 \mathrm{~nm}$ and $630 \mathrm{~nm}$ illumination were turned on at $151 \mathrm{~s}$ and off at $230 \mathrm{~s}$ and the LED illumination at $950 \mathrm{~nm}$ was turned on at $151 \mathrm{~s}$ and off at $291 \mathrm{~s}$.

\section{Results and Discussion}

The bulk current of R64039B was measured over time to determine the time dependence of the illumination effects and to analyse the de-trapping and transient bulk currents during and after optical excitation (Fig. 1). In all cases, an instantaneous increase in the bulk current was observed when the crystal was illuminated. An additional slow, yet minor exponential increase in the bulk

\footnotetext{
${ }^{\mathrm{i}}$ In this configuration, the crystal was first illuminated from the side, perpendicular to both the applied electric field and LED illumination, with a $75 \mathrm{~W}$ high stability xenon arc lamp (band pass of $1115 \pm 5 \mathrm{~nm})$. Minimal changes in the bulk current $\left(\sim 10^{-10} \mathrm{~A}\right)$ were observed when the crystal was illuminated at $\sim 1115 \mathrm{~nm}(1.11 \mathrm{eV})$, which is well below the band gap of the material.
} 
current over the duration of the illumination is also noted. Once the illumination was turned off, the current decreased within seconds (s). These results are in contrast to those of Adarsh et al. [16] who reported photocurrents that persisted after the illumination was turned off. We note, however, that these differences may be a result of the difference in dopant levels for the crystals examined in both studies.

The studies herein are somewhat similar in nature to photo-induced transient spectroscopy (PICTS) studies used to characterize trap levels in highly resistive semiconductors such as CdTe.[17] In general, the observed increases in bulk current shown in Fig. 1 can be attributed to both the generation of new charge carriers and the freeing of trapped charge in the crystal. CZT exhibits high absorption at energies equal to or above the band gap. The observed increases in bulk current that accompany illumination in this energy range can largely be attributed to the generation of new electron/hole pairs and phonons in the CZT bulk in addition to the freeing of trapped charges in the crystal, which may be located in mid-gap states.

We first examine the case where the energy range of illumination is above the band-gap (illumination at $630 \mathrm{~nm}$ and $470 \mathrm{~nm}$ ). Illumination in the above band-gap region is the basis for PICTS techniques. The higher absorptivity in this energy region creates an active volume of photogenerated carriers (electrons or holes, depending on the direction of the applied bias) that are injected from this region into the bulk of the crystal. In this case, transient currents are due to de-trapped holes or electrons. In Fig. 1 (A-E,) we see that there is an overall increase in the bulk current during illumination at $470 \mathrm{~nm}$ and $630 \mathrm{~nm}$. Further examination of the current evolution over time yields some insight into the de-trapping of charge carriers. The minor exponential increases in the steady-state photocurrent in both the forward (top surface is cathode) and reverse bias (top surface is anode) during illumination shown in Figs. 1 and 2 are indicative of detrapping of electrons and holes, respectively.[17, 18] These increases are followed by a sharp decrease in the bulk current once the illumination is turned off. Once the illumination is turned off, a negative peak is observed. The subsequent increase in current that follows this negative 
peak is the same as the initial increase in dark current following the application of the electric field. (refer to Fig. 2 in the supporting information).

In contrast, absorption of light at energies below the band-gap is minimal in CZT, resulting in nominal photocarrier generation. However, even with sub-band gap illumination at $950 \mathrm{~nm}$ (IR), an increase in the bulk current is observed (Fig. 1). This increase in bulk current during sub-band gap illumination is believed to be a result of freeing of trapped charges in the crystal during illumination. . The ability to free trapped charges with sub-band gap illumination of either the cathode or the anode has been previously suggested [8]. This concept is further supported by examining the exponential increase in current over time during illumination at $950 \mathrm{~nm}$ (Fig. 2), which is much more pronounced in both the forward and reverse bias than it is when the crystal is illuminated with above band-gap light (470 nm and $630 \mathrm{~nm})$. The IR illumination penetrates the entire crystal, such that holes and electrons are de-trapped throughout the bulk, reducing recombination sites and potentially increasing the mobility lifetime products of charge carriers. The combination of these effects increases the charge collection efficiency at the electrodes. The observed exponential increase of the current during IR illumination (950 nm, Figs. 1 and 2) reveals de-trapping of electrons and holes in both the forward (top surface is cathode) and reverse bias (top surface is anode) respectively.[17, 18] Furthermore, we note that when the crystal is forward biased, (Fig. 1, C-D) a transient current is observed following removal of IR illumination $(950 \mathrm{~nm})$. This slow, exponential decay is due to the re-trapping of electrons freed from deeperlevel traps or traps with larger capture cross sections.[17-19]

The increase in current during sub-band gap illumination of either the cathode or the anode presented herein occurs in conjunction with an observed polarization of the electric field toward the cathode. We note that this type of polarization effect has also been demonstrated to occur when CZT crystals are cooled below room temperature, although we have found that the degree of polarization at low temperature is much more significant with IR illumination.[8] Sellin et al.[7] reported that the observed polarization of the electric field on cooling at lower temperatures 
is due to a change in the charge injection barrier at the contacts that results from changes in the work function difference between CZT and Au. These changes can have deleterious effects on the charge collection efficiency at the read-out electrode. Our results are in contrast to these studies in that the light-induced polarization of the electric field is accompanied by an increase in the steady-state bulk current through the crystal. A possible explanation for this polarization was suggested where the increase in charge collection efficiency is due to increased lifetime of carriers in the crystal. The combination of the results from these two studies further indicates the possibility of two similar, yet separate mechanisms for the observed electric field enhancement.
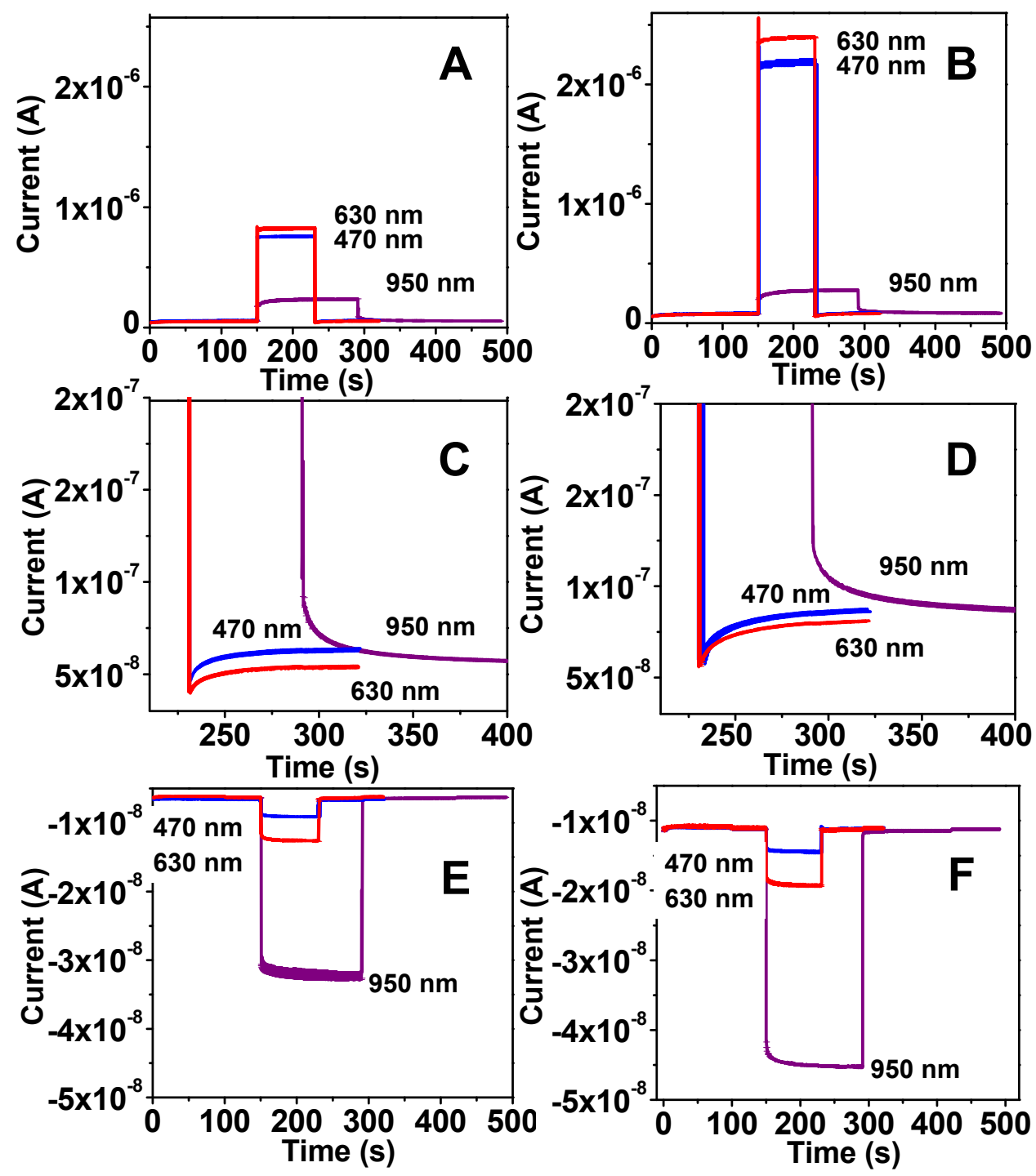
Figure 1. Steady-state bulk I-V measurements for CdZnTe crystal R64039B in planar configuration measured at applied electric fields of $\pm 78 \mathrm{~V} \mathrm{~mm}^{-1}$ (A,C,E) and $\pm 133 \mathrm{~V} \mathrm{~mm}^{-1}$ (B,D,F), with bias applied to the bottom contact (Cd-rich face). (A) $+350 \mathrm{~V}$ applied bias; (B) $+600 \mathrm{~V}$ applied bias, (C,D) zoom in of current when illumination is turned off, (E) $-350 \mathrm{~V}$ applied bias, (F) -600 V applied bias..
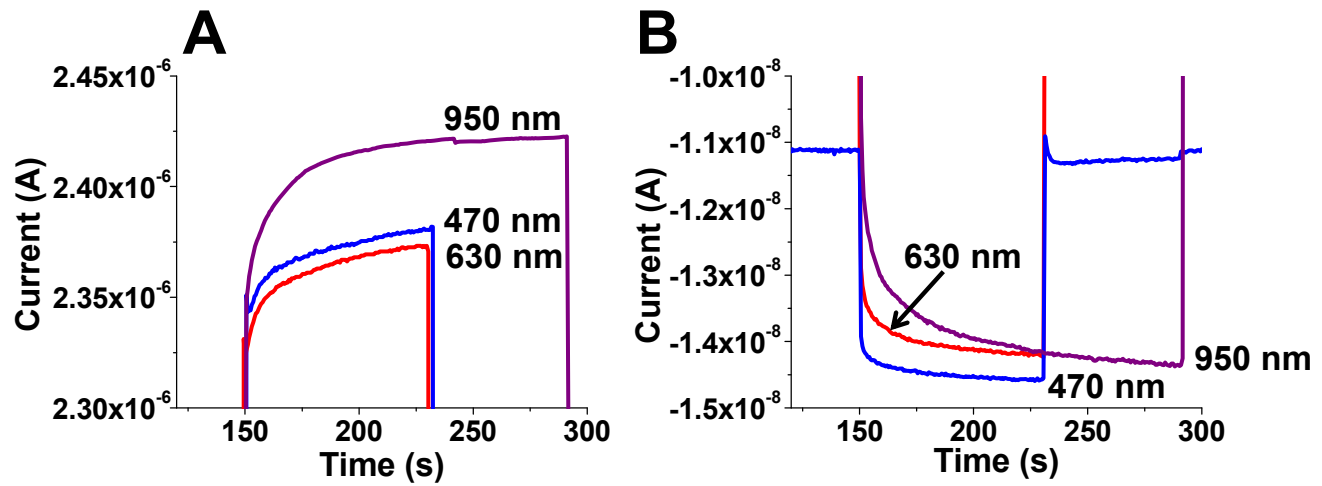

Figure 2. An enlargement of the current vs. time plot during application of illumination for CdZnTe crystal R64039B in planar configuration measured at applied electric fields of $\pm 133 \mathrm{~V}$ $\mathrm{mm}^{-1}$, with bias applied to the bottom contact (Cd-rich face). Curves are offset for comparison.

Current transients are not observed once the illumination at wavelengths above the band-gap is turned off. This indicates that the current increase is mostly due to photo-generated carriers and any de-trapped holes and electrons are quickly re-trapped in shallow level traps. We also note that the bulk current and the subsequent increase in the bulk current during illumination increases with the increasing applied electric field. These increases occur with the increasing applied electric field in all cases, except when the cathode is illuminated with IR light (see supporting information). In this one case, there is little dependence on the applied electric field.

In general, we find that the magnitude of the observed bulk currents exhibits a diode-like behaviour with higher currents observed when there is a positive bias on the bottom contact of the crystal. This behaviour is consistent with previous studies of CdTe and CZT [20] and is most 
likely due to the injection barriers at the $\mathrm{Au} / \mathrm{CZT}$ contacts. Each of the contacts has slightly different properties due to the interface composition (mainly $\mathrm{Cd}$ or Te, depending on crystal face). Current suppression in the reverse bias may also be due to the presence of a depletion region which, in turn, can affect the electric field distribution within the crystal.

To further compare the photoconductive response at various illumination wavelengths, we calculated the degree of asymmetry in the current enhancement during illumination $\left(\sim 78 \mathrm{~V} \mathrm{~mm}^{-1}\right.$, Table 1). This degree of asymmetry determination also provides insight into the optical absorption properties of the crystal. The degree of asymmetry is $\sim 1$ during IR illumination at 950 $\mathrm{nm}$ However, the increase in the degree of asymmetry in current enhancement for above bandgap illumination (values $>1$ for illumination at $470 \mathrm{~nm}$ and $630 \mathrm{~nm}$ ) is most likely due to absorption in the crystal, such that the light does not effectively free trapped charges throughout the entire volume of the crystal. It is generally accepted that the absorption coefficient for CdZnTe and CdTe is higher above the band gap, such that electron/hole pairs generated during illumination are confined closer to the top of the crystal (near the region of exposure). In this case, only one type of charge carrier is injected into the bulk, such that, when the crystal is reverse biased, the volume containing photo-induced carriers and de-trapped charges is small and located near the anode, and only holes are injected into the bulk. These holes may be subsequently re-trapped prior to collection at the cathode. When the crystal is forward biased, electrons are injected into the bulk. Consequently, the light does not fully penetrate the entire crystal, and the drift length of holes and electrons injected into the bulk of the crystal is proportional to the applied electric field density.[1] We also note that the observed current enhancement is proportional to the intensity of the light used (Fig. 3). In Fig. 3, the current increases as the illumination power increases, and slight changes in the curvature of the current evolution over time are indicative of slight increases in charge de-trapping. However, we do not observe transient hole or electron currents when the illumination intensity is increased. 


\section{A}
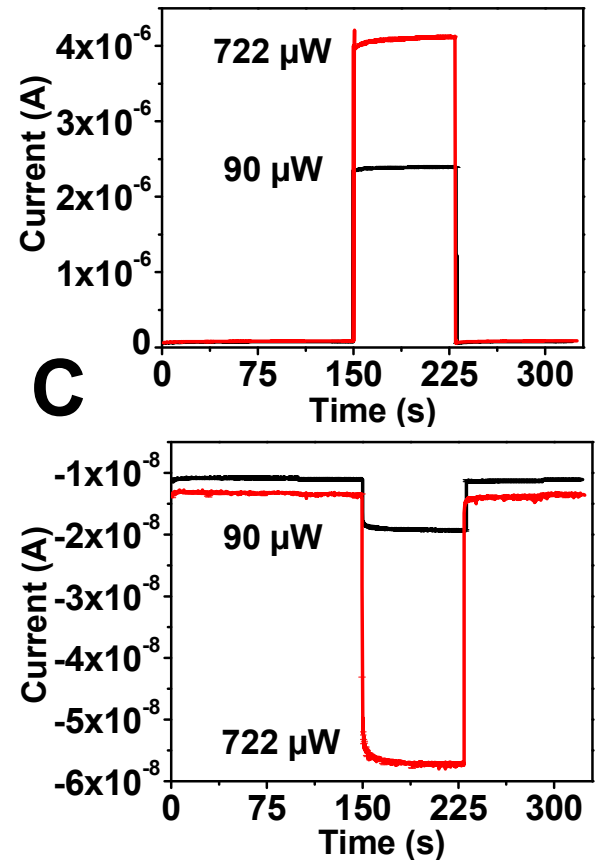

B
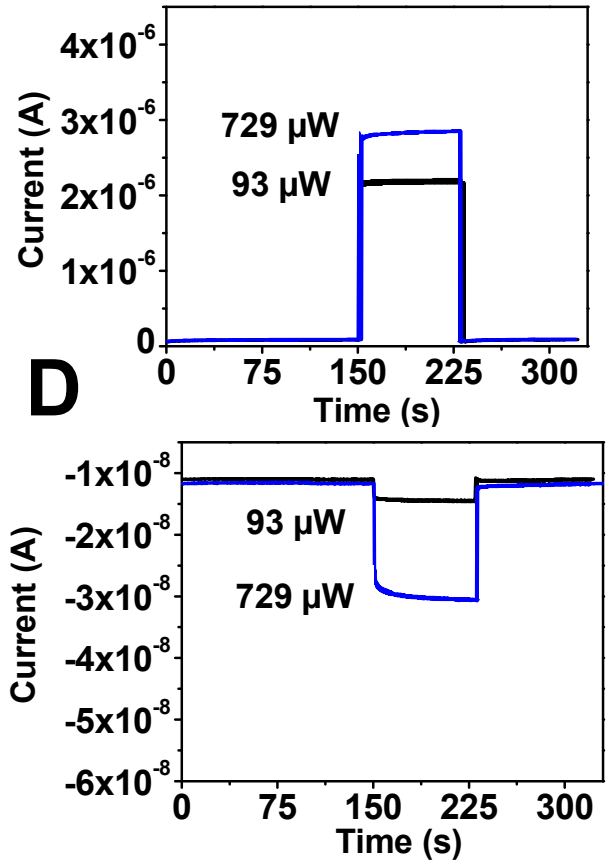

Figure 3. Steady-state bulk I-V measurements for R64039B CdZnTe crystal in planar configuration measured at applied electric field of $\pm 133 \mathrm{~V} \mathrm{~mm}^{-1}$ with bias applied to the bottom contact (Cd-rich face). (A) $+600 \mathrm{~V}$ applied bias, $630 \mathrm{~nm}$ illumination, (B) $-600 \mathrm{~V}$ applied bias, $630 \mathrm{~nm}$ illumination, (C) $+600 \mathrm{~V}$ applied bias, $470 \mathrm{~nm}$ illumination at, (D) $-600 \mathrm{~V}$ applied bias, $470 \mathrm{~nm}$ illumination.

Table 1. Results of studies with forward and reverse bias under the illumination of various wavelengths of light.

\begin{tabular}{|c|c|c|c|}
\hline & \multicolumn{2}{|c|}{ On/Off bulk current ratios } & \multirow{2}{*}{$\begin{array}{c}\text { Fwd/Rev ratio } \\
\text { degree of } \\
\text { asymmetry in } \\
\text { current } \\
\text { enhancement }\end{array}$} \\
\hline & Forward bias & Reverse bias & \\
\hline R64039B IR & 4.47 & 4.96 & 0.90 \\
\hline R64039B red & 16.36 & 2.00 & 8.17 \\
\hline R64039B blue & 17.15 & 3.10 & 5.54 \\
\hline YT-3-7-5 IR & 4.40 & 3.96 & 1.11 \\
\hline YT-3-7-5 red & 4.89 & 1.49 & 3.28 \\
\hline YT-3-7-5 blue & 8.31 & 1.52 & 5.46 \\
\hline
\end{tabular}

A comparison of the results from R64039B to bulk current-voltage (I-V) measurements of YT-3-7-8 with and without illumination (Figure 4) clearly show that this overall illumination 
response is not crystal dependent. Briefly, these measurements were conducted using a single pixel detector design, with the pixel electrode fabricated on the Te-rich surface of the CZT crystal (pixel diameter: $5 \mathrm{~mm}$ with $250 \mu \mathrm{m}$ gap). The bias voltage was applied to the top contacts, while the bottom contact was held at ground. LEDs were positioned at a fixed distance $(\sim 36 \mathrm{~mm})$ above the pixel surface during the measurements. The wavelengths and corresponding powers of the three LEDs that were used consisted of: a) $950 \mathrm{~nm}, 9.92 \mu \mathrm{W}$, b) $630 \mathrm{~nm}, 6.37 \mu \mathrm{W}$ and c) $470 \mathrm{~nm}$, $6.1 \mu \mathrm{W}$. The exposed surface area for this crystal was $2.01 \times 10^{-2} \mathrm{~cm}^{2}$, with an estimated 8 to $11 \%$ light transmission through the Au contacts.
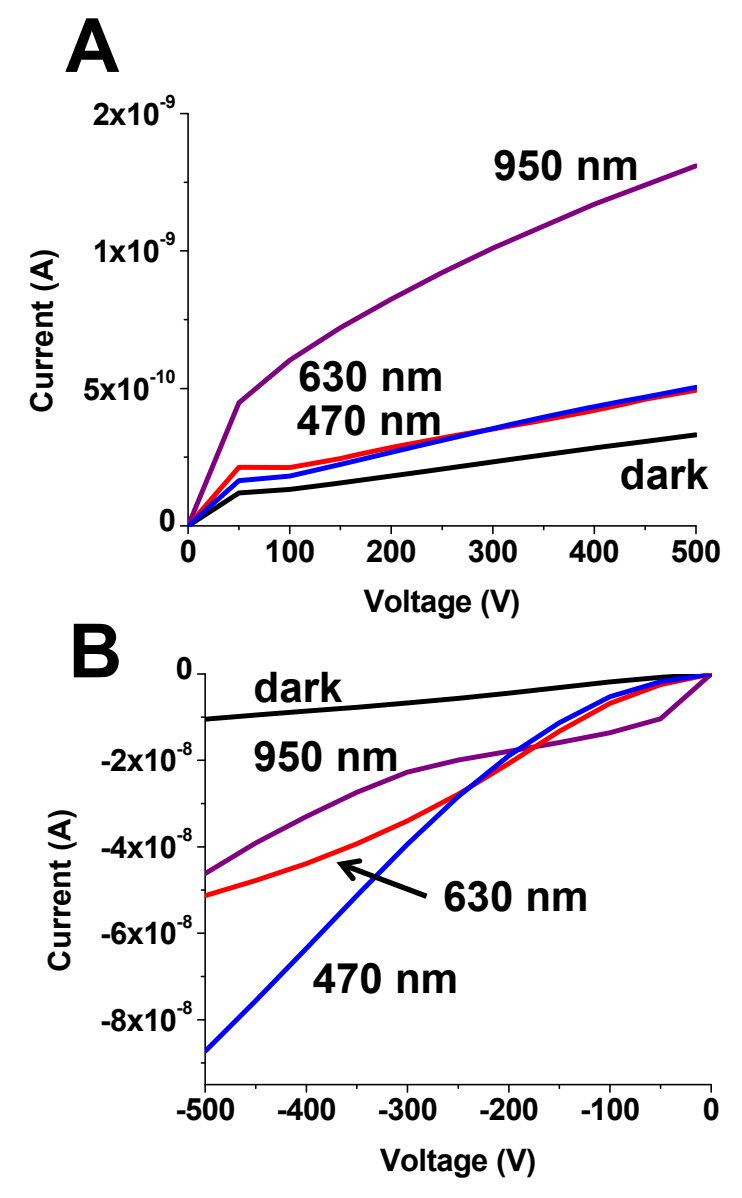

Figure 4. Bulk I-V measurements in (A) and (B) for the YT 3-7-8 crystal in single pixel configuration, with bias applied to the top contacts (Te-rich face). 
A comparison of the overall trends in the observed increases for both crystals yields insight into the charge transport dynamics and trap densities (Fig. 5). There are similarities in the bulk current response during illumination for these two crystals. There is a general increase in bulk current upon illumination, whereas the magnitude of that increase is wavelength, power, and electric field dependent (Figs. 1, 3 and 4). The degree of asymmetry in the current enhancement during illumination also provides some insight into the optical absorption properties and trap energies of these two crystals.

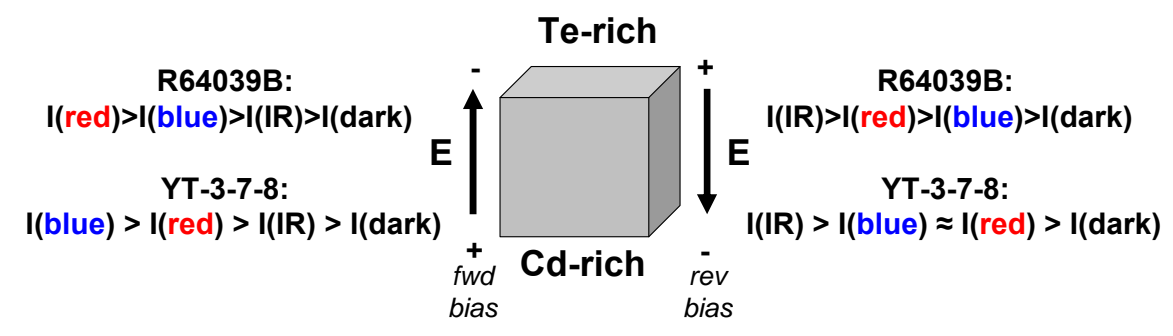

Figure 5. Diagram summarizing observed trends in bulk current changes as a function of illumination and direction of the applied electric field.

\section{Conclusions}

We have shown variations in the bulk currents in two different CdZnTe semiconductor crystals during and after exposure to LEDs of various wavelengths ranging from $470 \mathrm{~nm}$ to $950 \mathrm{~nm}$. The two crystals studied have similar added $\mathrm{Zn}$ content (in boule), but were grown under different conditions. The observed increase in bulk current during IR illumination is surprising and is attributed to the de-trapping of charge carriers which can, in turn, lead to a subsequent increase in carrier lifetime mobility products. Increases in bulk current during above band gap illumination (red and blue) are largely attributed to the generation of electron/hole pairs in addition to the detrapping of electrons and holes.

\section{Acknowledgements}

This manuscript has been authored by Savannah River Nuclear Solutions, LLC under Contract No. DE-AC09-08SR22470 with the U.S. Department of Energy. This work was supported by US 
DOE-NNSA, through the Office of Nonproliferation and Verification Research and Development

- NA-22 Grant No. DE-FG52-05NA27035. The United States Government retains and the

publisher, by accepting this article for publication, acknowledges that the United States

Government retains a non-exclusive, paid-up, irrevocable, worldwide license to publish or

reproduce the published form of this work, or allow others to do so, for United States

Government purposes.

\section{References}

[1] Schlesinger T E, Toney J E, Yoon H, E.Y. Lee, Burnett B A, Franks L and James R B 2001 Mater. Sci. Eng. R 32 103-89

[2] Amin N, Yamada A and Konagai M 2002 Jpn. J. Appl. Phys. 141 2834-41

[3] Mahawela P, Sivaraman G, Jeedigunta S, Gaduputi J, Ramalingam M, Subramanian S, Vakkalanka S, Ferekides C S and Morel D L 2005 Mater. Sci. Eng. B 116 283-91

[4] Verger L, Boitel M, Gentet M C, Hamelin R, Mestais C, Mongellaz F, Rustique J and Sanchez G 2001 Nucl. Instrum. Meth. A 458 297-309

[5] Duff M C, Hunter D B, Burger A, Groza M, Buliga V, Bradley J P, Graham G, Dai Z R, Teslich N, Black D R and Lanzirotti A 2009 J. Mater. Res. 24 1361-7

[6] Groza M, Krawczynski H, Garson A, Martin J W, Lee K, Li Q, Beilicke M, Cui Y L, Buliga V, Guo M S, Coca C and Burger A 2010 J. Appl. Phys. 107 023704-8

[7] Sellin P J, Prekas G, Franc J and Grill R 2010 Appl. Phys. Lett. 96 133509-3

[8] Washington A L, Teague L C, Duff M C, Burger A, Groza M and Buliga V (in press) J. Appl. Phys.

[9] Yao H W, Anderson R J and James R B 1997 Proc. SPIE 3115 62-8

[10] Yao H W, James R B and Erickson J C 1999 Proc. SPIE 3768 330-8

[11] Bale D S and Szeles C 2008 Phys. Rev. B 77035205

[12] Teague L C, Hawkins S A, Duff M C, Groza M, Buliga V and Burger A 2009 J. Electron. Mater. 38 1522-7

[13] Awadalla S A, Mackenzie J, Chen H, Redden B, Bindley G, Duff M C, Burger A, Groza M, Buliga V, Bradley J P, Dai Z R, Teslich N and Black D R 2010 J. Cryst. Growth 312 507-13

[14] Warekois E P, Mariano A N, Gatos H C and Lavine M C 1962 Journal of Applied Physics 33 690-\&

[15] Myers T H, Schetzina J F, Magee T J and Ormond R D 1983 J. Vac. Sci. Technol. A-Vac. Surf. Films 1 1598-603

[16] Adarsh K V, Sharon S, Mordechai S, Emil Z and Uri El H 2009 Light-Induced Reversible Shift and Control of the Bandgap of Bulk CdZnTe:V Crystals. OSA Technical Digest) p CMJ5

[17] Mathew X 2003 Sol. Energy Mater. Sol. Cells 76 225-42

[18] Baier N, Brambilla A, Feuillet G and Renet S 2006 Nucl. Instrum. Meth. A 563 155-8

[19] Rose A 1955 Phys. Rev. 97 1538-44 
[20] Duff M C, Hunter D B, Burger A, Groza M, Buliga V and Black D R 2008 Appl. Surf. Sci. 254 2889-92

\section{Supplementary Data}
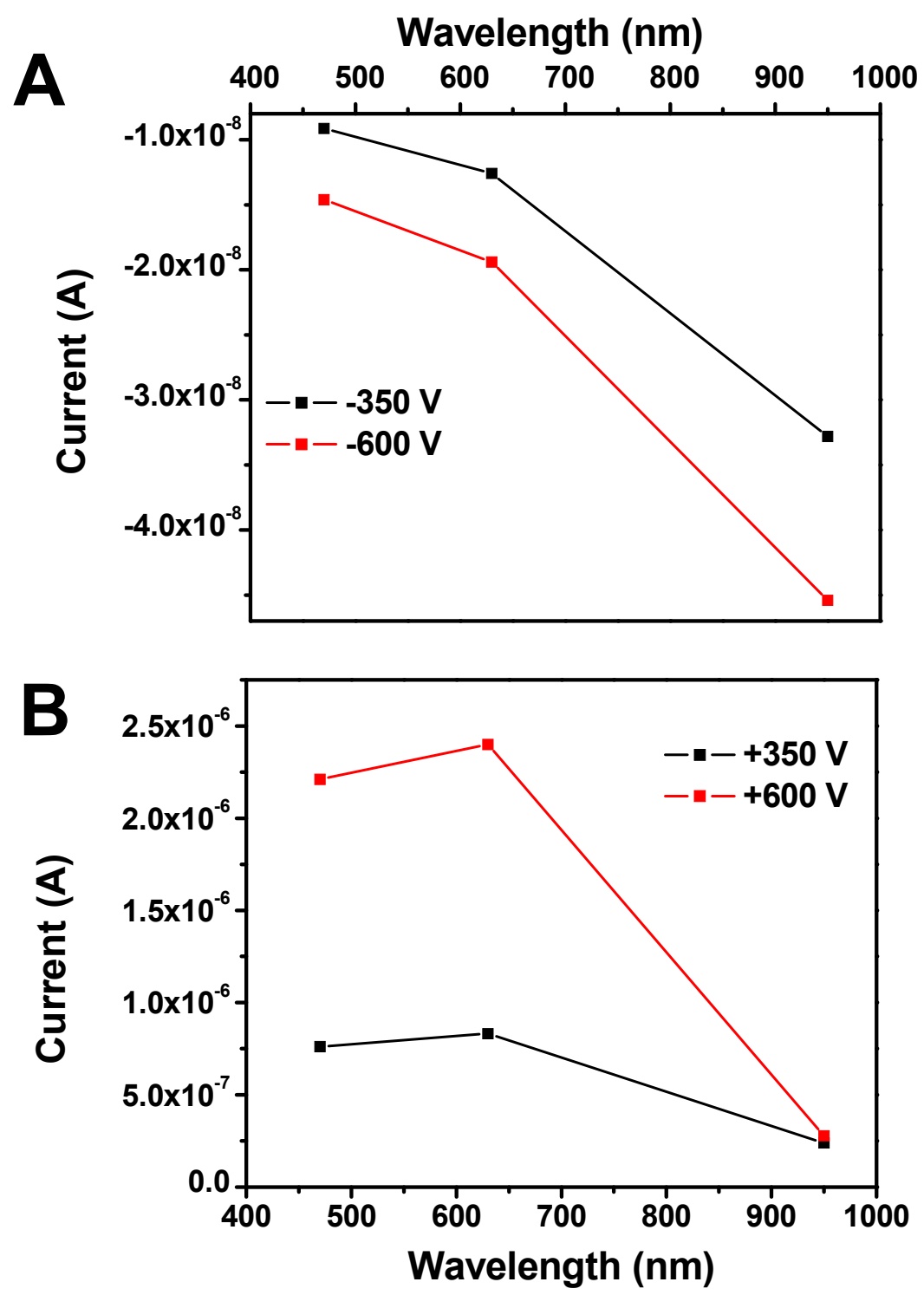

Figure S1. Average steady state bulk currents during illumination at various wavelengths for illumination of (A) anode and (B) cathode. The increase in the bulk current during illumination increases with the increasing applied electric field in all cases, except when the cathode is illuminated with IR light as shown here in (B). 


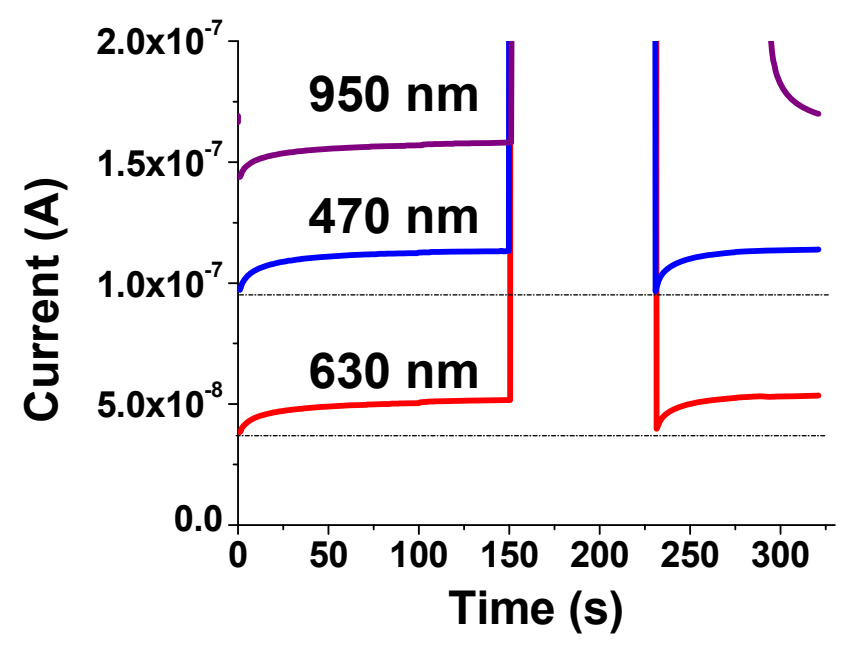

Figure S2. Steady-state bulk I-V measurements for CdZnTe crystal R64039B in planar configuration measured with $+350 \mathrm{~V}$ bias applied to the bottom contact (Cd-rich face). $1115 \mathrm{~nm}$ light turned on at 101 seconds (s); $470 \mathrm{~nm}$ and $630 \mathrm{~nm}$ illumination were turned on at $151 \mathrm{~s}$ and off at $230 \mathrm{~s} ; 950 \mathrm{~nm}$ illumination was turned on at $151 \mathrm{~s}$ and off at $291 \mathrm{~s}$. The subsequent increase in current that follows the negative peak in when the illumination at $470 \mathrm{~nm}$ and $630 \mathrm{~nm}$ is turned off is the same as the initial increase in dark current following the application of the electric field.

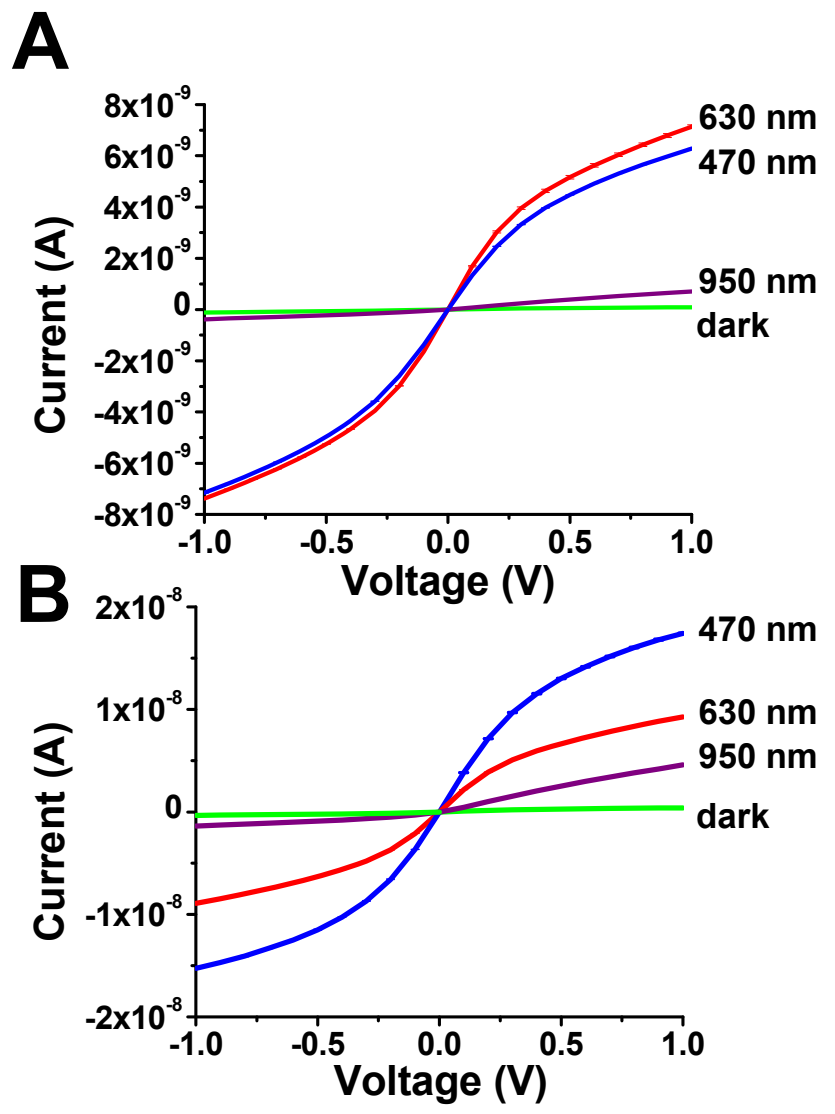


Figure S3. Surface current measured between pixel and guard contacts on the Te rich surface of the (a) R64039B and (b) YT 3-7-8 CdZnTe crystals. Each curve is an average of 3 IV measurements and error bars are shown. For both crystals, the surface current (surface resistivity) increases (decreases) during illumination, with the largest increases observed for surface illumination at $630 \mathrm{~nm}$ and $470 \mathrm{~nm}$. 